\title{
Łojasiewicz exponent of the gradient near the fiber
}

\author{
by Ha Huy Vui and Nguyen Hong Duc (Hanoi)
}

\begin{abstract}
It is well-known that if $r$ is a rational number from $[-1,0)$, then there is no polynomial $f$ in two complex variables and a fiber $f^{-1}\left(t_{0}\right)$ such that $r$ is the Eojasiewicz exponent of $\operatorname{grad}(f)$ near the fiber $f^{-1}\left(t_{0}\right)$. We show that this does not remain true if we consider polynomials in real variables. More exactly, we give examples showing that any rational number can be the Łojasiewicz exponent near the fiber of the gradient of some polynomial in real variables. The second main result of the paper is the formula computing the Łojasiewicz exponent of the gradient near a fiber of a polynomial in two real variables. In particular, this gives, in the case of two real variables, a way to tell whether a given value is an asymptotic critical value or not.
\end{abstract}

1. Introduction. Let $g: \mathbb{K}^{n} \rightarrow \mathbb{K}^{p}$ be a polynomial mapping, $\mathbb{K}=\mathbb{C}$ or $\mathbb{R}$. For an unbounded set $S \subset \mathbb{K}^{n}$, put

$\mathcal{L}_{\infty}\left(\left.g\right|_{S}\right):=\sup \left\{\nu \in \mathbb{R}: \exists C, R>0, \forall x \in S\left(\|x\| \geq R \Rightarrow\|g(x)\| \geq C\|x\|^{\nu}\right)\right\}$.

Let $f: \mathbb{K}^{n} \rightarrow \mathbb{K}$ be a polynomial function and $D_{\delta}=\left\{t:\left|t-t_{0}\right| \leq \delta\right\}$. Ha Huy Vui [H1] defined

$$
\mathcal{L}_{\infty}\left(f, t_{0}\right)=\lim _{\delta \rightarrow 0} \mathcal{L}_{\infty}\left(\left.\operatorname{grad} f\right|_{f^{-1}\left(D_{\delta}\right)}\right),
$$

or equivalently $([\mathrm{C}-\mathrm{K}],[\mathrm{Sk}])$

$$
\widetilde{\mathcal{L}}_{\infty}\left(f, t_{0}\right)=\inf _{\Phi} \frac{\operatorname{deg} \operatorname{grad} f \circ \Phi}{\operatorname{deg} \Phi},
$$

where $\Phi$ runs over the set of meromorphic functions in a neighborhood of infinity such that $\operatorname{deg} \Phi>0, \operatorname{deg}\left(f-t_{0}\right) \circ \Phi<0$. Following [R-S], we call them the Eojasiewicz exponent of the gradient of $f$ near the fiber $f^{-1}\left(t_{0}\right)$.

This exponent plays an important role in the study of polynomial mappings. According to a fundamental result of R. Thom [Th], there is a finite

2000 Mathematics Subject Classification: Primary 14R25; Secondary 32A20, 32S05, 14R25. Key words and phrases: Łojasiewicz exponent near the fiber, Puiseux expansion at infinity, Fedoryuk and Malgrange conditions, asymptotic critical value. 
subset $B(f)$ of $\mathbb{K}$, called the bifurcation set, such that the mapping

$$
f: \mathbb{K}^{n} \backslash f^{-1}(B(f)) \rightarrow \mathbb{K} \backslash B(f)
$$

defines a $C^{\infty}$ locally trivial fiber bundle. It is known that the set $\Sigma(f)$ of critical values of $f$ is a subset of $B(f)$ and in general it is not equal to $B(f)$. The points of $B_{\infty}(f)=B(f) \backslash \Sigma(f)$ are usually called critical values of singularities at infinity of $f$. It is important to be able to decide whether a given value in $\mathbb{K}$ belongs to $B_{\infty}(f)$. Although this problem has attracted attention of many specialists in singularity theory and algebraic geometry during the last twenty years $[\mathrm{C}-\mathrm{K}],[\mathrm{H} 1],[\mathrm{H}-\mathrm{L}],[\mathrm{P}]$, it is still open. We know the answer only for a number of particular cases.

It is easy to see that for any $n$, for $\mathbb{K}=\mathbb{C}$ or $\mathbb{K}=\mathbb{R}, \mathcal{L}_{\infty}\left(f, t_{0}\right)<0$ is always a necessary condition for $t_{0}$ to be in $B_{\infty}(f)$.

Assume $\mathbb{K}=\mathbb{C}$. If $n=2$, then a value $t_{0}$ belongs to $B_{\infty}(f)$ if and only if $\mathcal{L}_{\infty}\left(f, t_{0}\right)$ is negative. Moreover, if $\mathcal{L}_{\infty}\left(f, t_{0}\right)<0$ for some $t_{0}$ then $\mathcal{L}_{\infty}\left(f, t_{0}\right)<-1([\mathrm{H} 1],[\mathrm{C}-\mathrm{K}])$. The same holds for $n \geq 3$ if we assume that the polynomial $f$ has only isolated singularities at infinity $[\mathrm{P}]$.

In the general case, the above results are no longer true:

- There is a polynomial $f$ in three complex variables such that $\mathcal{L}_{\infty}\left(f, t_{0}\right)$ $<-1$ but $t_{0}$ is not a critical value of singularities at infinity for $f$ [P-Z].

- For $n \geq 3$, there is a polynomial $f$ of $n$ variables such that the set of all $t$ with $\mathcal{L}_{\infty}(f, t)<0$ is the whole $\mathbb{K}$, while the set of $t$ where $\mathcal{L}_{\infty}(f, t)<-1$ is always finite.

These facts lead to the following question which seems to be still open:

Is the set

$$
K_{\infty}(f):=\left\{t \in \mathbb{R}: \mathcal{L}_{\infty}(f, t)<-1\right\}
$$

equal to

$$
\tilde{K}_{\infty}(f):=\left\{t \in \mathbb{R}: \mathcal{L}_{\infty}(f, t)<0\right\}
$$

for any polynomial in two real variables?

We will show that for every rational number $\alpha$ there is a polynomial $f$ in $n \geq 2$ variables such that $\alpha=\mathcal{L}_{\infty}(f, t)$ for some $t$ (Theorem 2.1).

In particular, when $\mathbb{K}=\mathbb{R}$, the set $K_{\infty}(f)$ can be strictly included in $\tilde{K}_{\infty}(f)$, although, for $n=2$, both are finite.

Any point of $K_{\infty}(f)$ is called an asymptotic critical value of $f$. This notion appears in many problems of mathematics [K-M-P]. For example, the fact that $K_{\infty}(f)$ is finite plays an important role in the proof of the gradient conjecture [K-M-P].

We are interested in finding a simple way to decide whether a given value belongs to the set of asymptotic critical values or not. For polynomials in several complex variables, Jelonek and Kurdyka [J-K] gave an algorithm to compute this set. It turns out that this can also be done for polynomials in 
two variables, by using the Puiseux expansions at infinity (in the complex case) or their real approximations (in the real case) for the polar curve (Theorems 3.4 and 3.7).

\section{The Łojasiewicz exponent of the gradient near the fiber in the real case}

TheOREM 2.1. For every rational $\alpha$, there exists $f \in \mathbb{R}\left[x_{1}, \ldots, x_{n}\right]$ for which $\mathcal{L}_{\infty}(f, 0)=\alpha$.

Proof. We consider first the case $n=2$. It will be shown that $\mathcal{L}_{\infty}(f, 0)=$ $-2 q / p+2 m$ for the polynomial

$$
\begin{aligned}
f(x, y)= & \frac{1}{2 p+2 m+1} x^{2 p+2 m+1} y^{2 q}-\frac{2}{p+2 m+1} x^{p+2 m+1} y^{q} \\
& +\frac{1}{2 m+1} x^{2 m+1}+\frac{1}{2 m+3} x^{2 m+3} \\
& +\frac{1}{2 p+1} x^{2 p+1} y^{2 q+2 m}-\frac{2}{p+1} x^{p+1} y^{q+2 m}+x y^{2 m}+\frac{1}{3} x^{3} y^{2 m},
\end{aligned}
$$

where $p \geq q>0$ and $m \geq 0$ are integers. We see that

$$
\begin{aligned}
f_{x}(x, y)= & \frac{\partial f}{\partial x}(x, y)=\left[\left(x^{p} y^{q}-1\right)^{2}+x^{2}\right]\left(x^{2 m}+y^{2 m}\right), \\
f_{y}(x, y)= & \frac{2 q}{2 p+2 m+1} x^{2 p+2 m+1} y^{2 q-1}-\frac{2 q}{p+2 m+1} x^{p+2 m+1} y^{q-1} \\
& +\frac{2 q+2 m}{2 p+1} x^{2 p+1} y^{2 q+2 m-1}-\frac{2(q+2 m)}{p+1} x^{p+1} y^{q+2 m-1} \\
& +2 m x y^{2 m-1}+\frac{2 m}{3} x^{3} y^{2 m-1} .
\end{aligned}
$$

For the series $x=\varphi(y)=y^{-q / p}$, we have

$$
f_{x}(\varphi(y), y) \sim y^{-2 q / p+2 m} \text { and } f_{y}(\varphi(y), y) \sim y^{\beta}
$$

with $\beta \leq-2 q / p+2 m$. Thus

$$
\mathcal{L}_{\infty}(f, 0) \leq-2 q / p+2 m .
$$

For every $(x, y) \in \mathbb{R}^{2}, x^{2}+y^{2}=r^{2} \rightarrow \infty$. If $x^{p} y^{q}-1 \nrightarrow 0$ or $x \nrightarrow 0$ as $r \rightarrow \infty$ then

$$
\left|f_{x}(x, y)\right| \geq C r^{2 m} \geq C r^{-2 q / p+2 m} .
$$

Assume now that

$$
x^{p} y^{q}-1=\varepsilon(r) \rightarrow 0 \quad \text { and } \quad x \rightarrow 0 \quad \text { as } r \rightarrow \infty .
$$

We have

$$
|y|^{q}=\frac{1+\varepsilon(r)}{|x|^{p}} \leq r^{q} .
$$


Therefore

$$
x^{2} \geq C r^{-2 q / p} .
$$

Thus

$$
\left|f_{x}(x, y)\right| \geq C r^{-2 q / p+2 m} .
$$

Hence

$$
\mathcal{L}_{\infty}(f, 0) \geq-2 q / p+2 m .
$$

From (2.1) and (2.2), we get

$$
\mathcal{L}_{\infty}(f, 0)=-2 q / p+2 m \quad \forall p \geq q>0, m \geq 0 .
$$

Thus, for every rational $\alpha \geq-2$, there is an $f(x, y) \in \mathbb{R}[x, y]$ satisfying $\mathcal{L}_{\infty}(f, 0)=\alpha$.

Consider now the polynomial

$$
f(x, y)=x^{p} y^{q}-x,
$$

where $p>1, q>0$ are integers $\mathcal{L}_{\infty}(f, 0)=-\frac{p+q-1}{p-1}$. We have

$$
f_{x}(x, y)=p x^{p-1} y^{q}-1 \quad \text { and } \quad f_{y}(x, y)=q x^{p} y^{q-1} .
$$

Clearly

$$
\mathcal{L}_{\infty}(f, 0)=-\frac{p+q-1}{p-1}, \quad \forall p>1, q>0 .
$$

From (2.3) and (2.4), for every rational $\alpha$, there is an $f(x, y) \in \mathbb{R}[x, y]$ satisfying $\mathcal{L}_{\infty}(f, 0)=\alpha$.

Now we consider the general case. For every rational $\alpha$, we put

$$
F\left(x_{1}, x_{2}, \ldots, x_{n}\right)=f\left(x_{1}, x_{2}\right)+x_{3}^{k}+\cdots+x_{n}^{k},
$$

where $k>0$ is integer, $k \geq \alpha+1$ and $f \in \mathbb{R}[x, y]$ satisfies $\mathcal{L}_{\infty}(f, 0)=\alpha$. It is clear that $\mathcal{L}_{\infty}(F, 0)=\alpha$.

REMARK 2.2. A similar result (for the Łojasiewicz exponent at infinity) was given by E. A. Gorin (see [G]).

Let $f \in \mathbb{R}\left[x_{1}, \ldots x_{n}\right]$ and let $g: \mathbb{R}^{n} \rightarrow \mathbb{R}^{m}$ be a polynomial mapping. Let $t_{0} \in \mathbb{R}$. The authors of $[\mathrm{R}-\mathrm{S}]$ define

$$
\mathcal{L}_{\infty, f \rightarrow t_{0}}(g)=\sup \left\{\mathcal{L}_{\infty}\left(\left.g\right|_{f^{-1}(U)}\right): U \subset \mathbb{R}^{n} \text { is a neighborhood of } t_{0}\right\} .
$$

Analogously to Theorem 2.1 we can show

REmark 2.3. For every $\alpha \in \mathbb{Q}$ there exist $f \in \mathbb{R}\left[x_{1}, \ldots, x_{n}\right]$ and a polynomial mapping $g: \mathbb{R}^{n} \rightarrow \mathbb{R}^{m}$ such that $\mathcal{L}_{\infty, f \rightarrow 0}(g)=\alpha$. 
3. The Kojasiewicz exponent of the gradient near the fiber in the two variables case. We begin by recalling the definition of the Newton polygon relative to an arc and the process of sliding which were introduced by Kuo and Parusiński in [K-P].

If $\varphi(\tau)$ is a series of the form

$$
\varphi(\tau)=a_{0} \tau^{\alpha}+\text { terms of lower degree with } a_{0} \neq 0,
$$

then the number $\alpha$ is denoted by $\operatorname{deg} \varphi$.

Let $f: \mathbb{C}^{2} \rightarrow \mathbb{C}$ be a polynomial. For a series

$$
x=\varphi(y)=c_{1} y^{n_{1} / N}+c_{2} y^{n_{2} / N}+\cdots, \quad c_{i} \in \mathbb{C}^{2}, c_{1} \neq 0,
$$

we put

$$
M(X, Y)=f(X+\varphi(1 / Y), 1 / Y)=\sum_{i, j} c_{i j} X^{i} Y^{j / N} .
$$

For each $c_{i j} \neq 0$, let us plot a dot at $(i, j / N)$, called a Newton dot. The set of Newton dots is called the Newton diagram. They generate a convex hull, whose boundary is called the Newton polygon of $f$ relative to $\varphi$, to be denoted by $\mathbb{P}(f, \varphi)$ or $\mathbb{P}(M)$.

Assume that $x=\varphi(y)$ is not a Puiseux root at infinity of $f=0$. Then the $Y$-axis contains at least one $\operatorname{dot}$ of $M$. Let $\left(0, h_{M}\right)$ be the lowest Newton dot. We see that $h_{M}=-\operatorname{deg} f(\varphi(y), y)$.

By the highest Newton edge $H_{M}$ of $M$ we mean the edge of $\mathbb{P}(M)$ with one of extremities at $\left(0, h_{M}\right)$ and such that all Newton dots of $M$ lie on or above the line containing $H_{M}$. Let $\theta_{M}=\tan \nu$, where $\nu$ is the angle between $H_{M}$ and the $X$-axis. Note that if $(i, j / N)$ is a Newton dot of $M$ then $\theta_{M} i+j / N \geq h_{M}$, and $(i, j / N) \in H_{M}$ if and only if $\theta_{M} i+j / N=h_{M}$. If $x=\varphi(y)$ is a Puiseux root at infinity of $f=0$, we set $h_{M}=+\infty$ and $\theta_{M}=+\infty$.

We associate to $H_{M}$ the polynomial $\varepsilon_{M}(x):=\varepsilon(x, 1)$, where

$$
\varepsilon(X, Y)=\sum c_{i j} X^{i} Y^{j / N} \quad \text { with }(i, j / N) \in H_{M} .
$$

Lemma 3.1 ([H-D, Lemma 2.1]). Let $\widetilde{M}(X, Y)=M\left(X+c Y^{\theta}, Y\right)$.

(a) If $\theta>\theta_{M}$, then $h_{\widetilde{M}}=h_{M}$ and $\theta_{\widetilde{M}}=\theta_{M}$.

(b) If $\theta=\theta_{M}$ and $c$ is a non-zero root of $\varepsilon_{M}(x)$, then $h_{\widetilde{M}}>h_{M}$ and $\theta_{\widetilde{M}}>\theta_{M}$

(c) If $\theta=\theta_{M}$ and $\varepsilon_{M}(c) \neq 0$, then $h_{\widetilde{M}}=h_{M}$ and $\theta_{\widetilde{M}}=\theta_{M}$.

If $c$ is a non-zero root of $\varepsilon_{M}(x)$, the series $\varphi_{1}(y)=\varphi(y)+c y^{-\theta_{M}}$ will be called the sliding of $\varphi(y)$ along $f$. A recursive sliding $\varphi \rightarrow \varphi_{1} \rightarrow \cdots$ produces a limit, $\varphi_{\infty}$, where $\varphi_{\infty}(y)=\varphi_{i}(y)$ if $f\left(\varphi_{i}(y), y\right)=0$. The series $\varphi_{\infty}$ is a Puiseux root at infinity of $f=0$ (see [W]) and will be called a final result of sliding $\varphi$ along $f$. 
Lemma 3.2 ([H-D, Lemma 2.2]). Let $f, g: \mathbb{C}^{2} \rightarrow \mathbb{C}$ be two polynomials. For a series $x=\varphi(y)$, we put

$$
\begin{aligned}
M(X, Y) & =f(X+\varphi(1 / Y), 1 / Y), \\
N(X, Y) & =g(X+\varphi(1 / Y), 1 / Y) .
\end{aligned}
$$

We have

(a) if $\theta_{M}>\theta_{N}$, then $\operatorname{deg} g\left(\varphi_{\infty}(y), y\right)=\operatorname{deg} g(\varphi(y), y)$;

(b) if $\theta_{M}=\theta_{N}$, then $\operatorname{deg} g\left(\varphi_{\infty}(y), y\right) \leq \operatorname{deg} g(\varphi(y), y)$,

where $x=\varphi_{\infty}(y)$ is a final result of sliding $\varphi$ along $f$.

Let us consider a series $x=\lambda(y)$ of the form

$$
x=\lambda(y)=a_{1} y^{\alpha_{1}}+a_{2} y^{\alpha_{2}}+\cdots+a_{s-1} y^{\alpha_{s-1}}+a_{s} y^{\alpha_{s}}+\cdots
$$

where $\alpha_{1}>\alpha_{2}>\cdots$.

If $a_{1}, \ldots, a_{s-1} \in \mathbb{R}$ and $a_{s} \notin \mathbb{R}$, following Kuo [K] we put

$$
\lambda^{\mathbb{R}}(y):=a_{1} y^{\alpha_{1}}+a_{2} y^{\alpha_{2}}+\cdots+a_{s-1} y^{\alpha_{s-1}}+c y^{\alpha_{s}},
$$

where $c$ is a generic real number. We call $\lambda^{\mathbb{R}}(y)$ the real approximation of $\lambda(y)$.

Lemma 3.3 ([H-D, Lemma 2.3]). Let $f, g: \mathbb{R}^{2} \rightarrow \mathbb{R}$ be polynomials. For a series $x=\varphi(y)$, we put

$$
\begin{aligned}
M(X, Y) & =f(X+\varphi(1 / Y), 1 / Y), \\
N(X, Y) & =g(X+\varphi(1 / Y), 1 / Y) .
\end{aligned}
$$

Let $x=\varphi_{\infty}(y)$ be a final result of sliding $\varphi$ along $f$ and $\varphi_{\infty}^{\mathbb{R}}(y)$ be the real approximation of $\varphi_{\infty}(y)$. We have

(a) if $\theta_{M}>\theta_{N}$, then $\operatorname{deg} g\left(\varphi_{\infty}^{\mathbb{R}}(y), y\right)=\operatorname{deg} g(\varphi(y), y)$;

(b) if $\theta_{M}=\theta_{N}$, then $\operatorname{deg} g\left(\varphi_{\infty}^{\mathbb{R}}(y), y\right) \leq \operatorname{deg} g(\varphi(y), y)$.

In particular for $g=f$, we have $\operatorname{deg} f\left(\varphi_{\infty}^{\mathbb{R}}(y), y\right) \leq \operatorname{deg} f(\varphi(y), y)$.

TheOREM 3.4. Let $f$ be a polynomial in two complex variables $(x, y)$. Assume that $f$ is monic in $x$, and $t_{0} \in \mathbb{C}$. Let $x=x_{i}(y), i=1, \ldots, d-1$, be the Puiseux expansions at infinity of $f_{x}(x, y)=0$. Put

$$
V\left(f, t_{0}\right)=\left\{x_{i}(y): \operatorname{deg}\left(f\left(x_{i}(y), y\right)-t_{0}\right)<0\right\} .
$$

If $\mathcal{L}_{\infty, f \rightarrow t_{0}}(\operatorname{grad} f)<0$, then $V\left(f, t_{0}\right) \neq \emptyset$ and

$$
\mathcal{L}_{\infty, f \rightarrow t_{0}}(\operatorname{grad} f)=\min _{i}\left\{\operatorname{deg}\left(f\left(x_{i}(y), y\right)-t_{0}\right)-1: x_{i}(y) \in V\left(f, t_{0}\right)\right\} .
$$

Proof. Let $x=\varphi(y)$ be any series satisfying

$$
\operatorname{deg}\left(f(\varphi(y), y)-t_{0}\right)<0, \quad \operatorname{deg} \operatorname{grad} f(\varphi(y), y)<0 .
$$


We put

$$
\begin{aligned}
M(X, Y) & =f(X+\varphi(1 / Y), 1 / Y)-t_{0}=\sum_{i, j} c_{i j} X^{i} Y^{j / N}, \\
P(X, Y) & =f_{x}(X+\varphi(1 / Y), 1 / Y), \\
Q(X, Y) & =f_{x}(X+\varphi(1 / Y), 1 / Y)+c f_{y}(X+\varphi(1 / Y), 1 / Y), \\
R(X, Y) & =f_{x}(X+\varphi(1 / Y), 1 / Y)+c\left(f(X+\varphi(1 / Y), 1 / Y)-t_{0}\right),
\end{aligned}
$$

where $c$ is a generic number. Then

$$
\begin{aligned}
P(X, Y) & =\frac{\partial M}{\partial X}(X, Y), \\
Q(X, Y) & =\frac{\partial M}{\partial X}(X, Y)-c\left(\varphi^{\prime}\left(\frac{1}{Y}\right) \frac{\partial M}{\partial X}(X, Y)+Y^{2} \frac{\partial M}{\partial Y}(X, Y)\right), \\
R(X, Y) & =\frac{\partial M}{\partial X}(X, Y)-c M(X, Y) .
\end{aligned}
$$

Claim. $\theta_{P}>0, \theta_{P} \geq \theta_{Q}$ and $\theta_{P} \geq \theta_{R}$.

Note that if $(i, j / N)$ is a Newton dot of $P$ then $\theta_{P} i+j / N \geq h_{P}$. Since the point $(d-1,0)$ is a Newton dot of $P, \theta_{P}(d-1)+0 \geq h_{P}$. Hence $\theta_{P}>0$.

Let $(\alpha, \beta)$ be the second extremity of $H_{Q}$. We will show that $\theta_{P} \alpha+\beta$ $\geq h_{P}$. In fact:

- If $(\alpha, \beta)$ is a Newton dot of $P$, then $\theta_{P} \alpha+\beta \geq h_{P}$.

- If $(\alpha, \beta)$ is a Newton dot of $\varphi^{\prime}(1 / Y) \frac{\partial M}{\partial X}(X, Y)$, then $(\alpha, \beta-s)$ is a dot of $P$, where $s \geq 0$ since $\operatorname{deg} \varphi \leq 1$. Therefore

$$
\theta_{P} \alpha+(\beta-s) \geq h_{P}
$$

Thus

$$
\theta_{P} \alpha+\beta \geq h_{P}
$$

- If $(\alpha, \beta)$ is a Newton dot of $Y^{2} \frac{\partial M}{\partial Y}(X, Y)$, then $(\alpha-1, \beta-1)$ is a dot of $P$. Therefore

$$
\theta_{P}(\alpha-1)+(\beta-1) \geq h_{P}
$$

Thus $\theta_{P} \alpha+\beta \geq h_{P}$ since $\theta_{P}>0$.

Since the point $\left(0, h_{P}\right)$ is a Newton dot of $Q, h_{P} \geq h_{Q}$. Therefore

$$
\theta_{P} \alpha+\beta \geq h_{P} \geq h_{Q}=\theta_{Q} \alpha+\beta \text {. }
$$

Hence $\theta_{P} \geq \theta_{Q}$.

Analogously, we can show that $\theta_{P} \geq \theta_{R}$.

Now, using the claim and Lemma 3.2, we get

$$
\operatorname{deg}\left(f_{x}\left(\varphi_{\infty}(y), y\right)+c f_{y}\left(\varphi_{\infty}(y), y\right)\right) \leq \operatorname{deg}\left(f_{x}(\varphi(y), y)+c f_{y}(\varphi(y), y)\right)
$$


and

$$
\begin{aligned}
\operatorname{deg}\left(f_{x}\left(\varphi_{\infty}(y), y\right)+c\left(f\left(\varphi_{\infty}(y), y\right)-t_{0}\right)\right) & \\
& \leq \operatorname{deg}\left(f_{x}(\varphi(y), y)+c\left(f(\varphi(y), y)-t_{0}\right)\right),
\end{aligned}
$$

where $\varphi_{\infty}(y)$ is a final result of sliding $\varphi(y)$ along $f_{x}$. Therefore

$$
\begin{aligned}
\operatorname{deg}\left(f\left(\varphi_{\infty}(y), y\right)-t_{0}\right)-1 & =\operatorname{deg} \frac{d f\left(\varphi_{\infty}(y), y\right)}{d y}=\operatorname{deg} f_{y}\left(\varphi_{\infty}(y), y\right) \\
& \leq \operatorname{deg}(\operatorname{grad} f(\varphi(y), y))
\end{aligned}
$$

and

$$
\operatorname{deg}\left(f\left(\varphi_{\infty}(y), y\right)-t_{0}\right) \leq \operatorname{deg}\left(f_{x}(\varphi(y), y)+c f(\varphi(y), y)\right)<0 .
$$

Thus $V\left(f, t_{0}\right) \neq \emptyset$ and

$$
\mathcal{L}_{\infty, f \rightarrow t_{0}}(\operatorname{grad} f) \geq \min _{i}\left\{\operatorname{deg}\left(f\left(x_{i}(y), y\right)-t_{0}\right)-1: x_{i}(y) \in V\left(f, t_{0}\right)\right\} .
$$

On the other hand, the inequality

$$
\mathcal{L}_{\infty, f \rightarrow t_{0}}(\operatorname{grad} f) \leq \min _{i}\left\{\operatorname{deg}\left(f\left(x_{i}(y), y\right)-t_{0}\right)-1: x_{i}(y) \in V\left(f, t_{0}\right)\right\}
$$

is always satisfied. Hence

$$
\mathcal{L}_{\infty, f \rightarrow t_{0}}(\operatorname{grad} f)=\min _{i}\left\{\operatorname{deg}\left(f\left(x_{i}(y), y\right)-t_{0}\right)-1: x_{i}(y) \in V\left(f, t_{0}\right)\right\} .
$$

REMARK 3.5. This result is implicitly contained in [K-P] (see also [H2] for a different proof).

Corollary 3.6 ([G-S, Theorem 3.1]). Let $f(x, y)$ be a polynomial in two complex variables and $d=\operatorname{deg} f>2$. If $\mathcal{L}_{\infty, f \rightarrow t_{0}}(\operatorname{grad} f)<0$, then

$$
\mathcal{L}_{\infty, f \rightarrow t_{0}}(\operatorname{grad} f) \leq-1-\frac{1}{d-2} .
$$

Proof. The proof goes along the same lines as in [G-S]. Let $x=x_{i}(y)$, $i=1, \ldots, d-1$, be the Puiseux expansions at infinity of $f_{x}(x, y)=0$. Assume that $\mathcal{L}_{\infty, f \rightarrow t_{0}}(\operatorname{grad} f)<0$. By Theorem 3.4 there exists $i_{0} \in\{1, \ldots, d-1\}$ such that

$$
\mathcal{L}_{\infty, f \rightarrow t_{0}}(\operatorname{grad} f)=\operatorname{deg}\left(f\left(x_{i_{0}}(y), y\right)-t_{0}\right)-1
$$

and

$$
\operatorname{deg}\left(f\left(x_{i_{0}}(y), y\right)-t_{0}\right)<0 .
$$

Let $Q \in \mathbb{C}[\tau, y]$ be the resultant $Q(\tau, y)=\operatorname{Res}_{x}\left(f-\tau, f_{x}\right)$. Denote by $\mathbb{P}$ the Newton polygon of $Q(\tau, y)$. Then

$$
\mathbb{P} \subset \operatorname{conv}\{(0,0) ;(d-1,0) ;(0, d(d-1))\}
$$

and

$$
Q(\tau, y)=\prod_{i=1}^{d-1}\left(f\left(x_{i}(y), y\right)-\tau\right)
$$


From (3.1), analogously to [G-S, Lemma 3.3] we obtain

$$
\operatorname{deg} f\left(x_{i_{0}}(y), y\right)=-\frac{j_{2}-j_{1}}{i_{2}-i_{1}},
$$

where the segment $\left[\left(i_{1}, j_{1}\right),\left(i_{1}, j_{2}\right)\right] \subset \mathbb{P}$ is such that $i_{1}, i_{2}, j_{1}, j_{2} \in \mathbb{Z}, 0 \leq$ $i_{1}<i_{2}, 0 \leq j_{1}<j_{2}$. By (3.2) we see $i_{2}-i_{1} \leq d-2$. Therefore

$$
\operatorname{deg} f\left(x_{i_{0}}(y), y\right) \leq-\frac{1}{d-2} .
$$

Thus

$$
\mathcal{L}_{\infty, f \rightarrow t_{0}}(\operatorname{grad} f) \leq-1-\frac{1}{d-2} .
$$

Theorem 3.7. Let $f: \mathbb{R}^{2} \rightarrow \mathbb{R}$ be a monic polynomial in $x$. Let $t_{0} \in \mathbb{R}$. Let $x=x_{i}(y), i=1, \ldots, d-1$, be the Puiseux expansions at infinity of $f_{x}(x, y)=0$ and $x_{i}^{\mathbb{R}}(y)$ be the real approximation of $x_{i}(y)$. Put

$$
V_{\mathbb{R}}\left(f, t_{0}\right)=\left\{x_{i}(y): \operatorname{deg}\left(f\left(x_{i}^{\mathbb{R}}(y), y\right)-t_{0}\right)<0\right\} .
$$

If $\mathcal{L}_{\infty, f \rightarrow t_{0}}(\operatorname{grad} f)<0$, then $V_{\mathbb{R}}\left(f, t_{0}\right) \neq \emptyset$ and

$$
\mathcal{L}_{\infty, f \rightarrow t_{0}}(\operatorname{grad} f)=\min _{i}\left\{\operatorname{deg} \operatorname{grad} f\left(x_{i}^{\mathbb{R}}(y), y\right): x_{i}(y) \in V_{\mathbb{R}}\left(f, t_{0}\right)\right\} .
$$

Proof. Let $x=\varphi(y)$ be any real series satisfying

$$
\operatorname{deg}\left(f(\varphi(y), y)-t_{0}\right)<0, \quad \operatorname{deg} \operatorname{grad} f(\varphi(y), y)<0 .
$$

We put

$$
\begin{aligned}
M(X, Y) & =f(X+\varphi(1 / Y), 1 / Y)-t_{0}=\sum_{i, j} c_{i j} X^{i} Y^{j / N}, \\
P(X, Y) & =f_{x}(X+\varphi(1 / Y), 1 / Y), \\
Q(X, Y) & =f_{x}(X+\varphi(1 / Y), 1 / Y)+c f_{y}(X+\varphi(1 / Y), 1 / Y), \\
R(X, Y) & =f_{x}(X+\varphi(1 / Y), 1 / Y)+c\left(f(X+\varphi(1 / Y), 1 / Y)-t_{0}\right),
\end{aligned}
$$

where $c$ is a generic number.

Using the Claim in the proof of Theorem 3.4, we get $\theta_{P} \geq \theta_{Q}$ and $\theta_{P} \geq \theta_{R}$.

Now, the proof repeats that of Theorem 3.4 with the only exception that instead of Lemma 3.2 we use Lemma 3.3.

REMARK 3.8. The following conditions on the behavior at infinity of the gradient of maps appear in many problems. Let $f: \mathbb{K}^{n} \rightarrow \mathbb{K}$ be a polynomial and $t_{0} \in \mathbb{K}$.

(1) We say that $t_{0}$ satisfies the Fedoryuk condition (F) if $\mathcal{L}_{\infty, f \rightarrow t_{0}}(\operatorname{grad} f)$ $\geq 0$, i.e. for every sequence $\left\{z_{m}\right\} \subset \mathbb{K}^{n}$ with $z_{m} \rightarrow \infty$ and $f\left(z_{m}\right) \rightarrow t_{0}$ we have $\operatorname{grad} f\left(z_{m}\right) \nrightarrow 0$.

(2) We say $t_{0}$ satisfies the Malgrange condition $(\mathrm{M})$ if $\mathcal{L}_{\infty, f \rightarrow t_{0}}(\operatorname{grad} f)$ $\geq-1$, i.e. for every sequence $\left\{z_{m}\right\} \subset \mathbb{K}^{n}$ with $z_{m} \rightarrow \infty$ and $f\left(z_{m}\right) \rightarrow$ $t_{0}$ we have $\left\|z_{m}\right\|\left\|\operatorname{grad} f\left(z_{m}\right)\right\| \nrightarrow 0$. 
(3) We say that $t_{0}$ is an asymptotic critical value if it does not satisfy the Malgrange condition.

Theorem 3.7 gives us a simple way to check whether a given value $t_{0} \in \mathbb{R}$ satisfies $(\mathrm{F})$ or $(\mathrm{M})$ or not. In fact, with the notation of Theorem 3.7, it is enough to compute

$$
\min _{i}\left\{\operatorname{deg} \operatorname{grad} f\left(x_{i}^{\mathbb{R}}(y), y\right): x_{i}(y) \in V_{\mathbb{R}}\left(f, t_{0}\right)\right\},
$$

and to compare it with 0 or -1 .

Acknowledgments. The authors would like to thank the referee for his precious comments and suggestions which improved the presentation of this paper.

\section{References}

[C-K] J. Chądzyński and T. Krasiński, The gradient of a polynomial at infinity, Kodai Math. J. 26 (2003), 317-339.

[G] E. A. Gorin, Asymptotic properties of polynomials and algebraic functions of several variables, Russian Math. Survyes 16 (1961), 93-119.

[G-S] J. Gwoździewicz and S. Spodzieja, The Eojasiewicz gradient inequality in a neighbourhood of the fibre, Ann. Polon. Math. 87 (2005), 151-163.

[H1] Hà Huy Vui, Nombres de Eojasiewicz et singularités à l'infini des polynômes de deux variables complexes, C. R. Acad. Sci. Paris 331 (1990), 429-432.

[H2] - Infimum of polynomials and singularity at infinity, in: Nonconvex Optim. Appl. 53, Kluwer, 2001, 187-204.

[H-L] Hà Huy Vui and Lê Dũng Tráng, Sur la topologie des polynômes complexes, Acta Math. Vietnam. 9 (1984), 21-32.

[H-D] Hà Huy Vui and Nguyen Hong Duc, On the Eojasiewicz exponent near the fibre of polynomial mappings, Ann. Polon. Math. 94 (2008), 43-52.

[J-K] Z. Jelonek and K. Kurdyka, On asymptotic critical values of a complex polynomial, J. Reine Angew. Math. 565 (2003), 1-11.

[K] T.-C. Kuo, Computation of Lojasiewicz exponent of $f(x, y)$, Comment. Math. Helv. 49 (1974), 201-213.

[K-P] T.-C. Kuo and A. Parusiński, Newton polygon relative to an arc, in: Real and Complex Singularities, Chapman \& Hall/CRC Res. Notes Math. 412 (2000), 76-93.

[K-M-P] K. Kurdyka, T. Mostowski and A. Parusiński, Proof of the gradient conjecture of R. Thom, Ann. of Math. 152 (2000), 763-792.

[P] A. Parusiński, On the bifurcation set of complex polynomial with isolated singularities at infinity, Compos. Math. 97 (1995), 369-384.

[P-Z] L. Paunescu and A. Zaharia, On the Eojasiewicz exponent at infinity for polynomial functions, Kodai Math. J. 20 (1997), 269-274.

[R-S] T. Rodak and S. Spodzieja, Eojasiewicz exponent near the fibre of a mapping, Faculty of Math. and Comput. Sci., Univ. of Łódź, preprint 03/2005.

[Sk] G. Skalski, On the Eojasiewicz exponent near the fibre of a polynomial, Bull. Polish Acad. Sci. Math. 52 (2004), 231-236. 
[Th] R. Thom, Ensembles et morphismes stratifiés, Bull. Amer. Math. Soc. 75 (1969), 249-312.

[W] R. Walker, Algebraic Curves, Princeton Univ. Press, 1950.

Institute of Mathematics

18 Hoang Quoc Viet Road

Cau Giay District

10307, Hanoi, Vietnam

E-mail: hhvui@math.ac.vn

nhduc@math.ac.vn

Received 24.3.2008

and in final form 3.4.2009

(1866) 
